\section{Saúde e nutrição de crianças de áreas urbanas da Zona da Mata Meridional de Pernambuco: resultados preliminares de um estudo de coorte}

\section{Child health and nutrition in urban areas in the South of Pernambuco: preliminary results of a cohort study}

Pedro Israel Cabral de Lira 1 Marilia de Carvalho Lima 2

Gisélia Alves Pontes da Silva 3

Sylvia de Azevedo Mello Romani 4 Sophie Helena Eickmann 5 Maria Luiza Martins Alessio 6 Malaquias Batista Filho 7

Claude Louis Leger 8 Sharon Rebeca Huttly 9 Ann Ashworth 10

1,4,7 Departamento de Nutrição. Universidade Federal de Pernambuco. Rua Prof. Nelson Chaves, s/n. Cidade Universitária. Recife, PE, Brasil. CEP: 50.670-901

2,3,5 Departamento Materno-Infantil. Universidade Federal de Pernambuco. 6 Departamento de Fisiologia e Farmacologia. Universidade Federal de Pernambuco, Brasil.

${ }^{8}$ Laboratoire de Nutrition Humaine et Athérogènese de la Université Montpellier I, France

9,10 London School of Hygiene and Tropical Medicine. London, UK

\section{Resumo}

Objetivos: apresentar as características socioeconômicas e demográficas, o perfil nutricional de crianças ao nascer e aos 12 meses de vida, além dos dados longitudinais sobre aleitamento, diarréia e situação vacinal durante o primeiro ano de vida.

Métodos: uma amostra de 652 recém-nascidos foi recrutada de setembro de 1997 a agosto de 1998 e acompanhada durante os primeiros 18 meses de vida. Essas crianças residiam nas áreas urbanas de quatro municípios da zona da mata meridional de Pernambuco. A coleta de dados foi realizada através de visitas domiciliares.

Resultados: cerca de $60 \%$ das famílias tinham uma renda per capita $\leq 1 / 2$ salário mínimo e $41 \%$ das mães referiam menos de quatro anos de escolaridade. A mediana do aleitamento materno exclusivo e total foi de 0 dias e 94 dias, respectivamente. A incidência da diarréia foi de dois episódios/criança/ano nos primeiros 12 meses de vida e a prevalência de déficit peso/idade e comprimento/idade (<-2 escores z) aos 12 meses foram de 6,8\% e $11 \%$, respectivamente. Apenas $66 \%$ das crianças aos 12 meses tinham completado o esquema vacinal.

Conclusões: o desenvolvimento desta pesquisa prospectiva contribuirá para uma maior compreensão dos problemas de saúde e nutrição da população infantil e para o adequado planejamento de intervenções na área.

Palavras-chave Bem-estar do lactente, Nutrição infantil 


\section{Introdução}

A maioria ou mesmo a quase totalidade dos estudos sobre a situação de saúde e nutrição de populações dos países pobres utilizam a abordagem seccional, baseada em amostras probabilísticas ou não. Mesmo produzindo resultados interessantes, na medida em que definem linhas de base e podem elencar um conjunto hipotético de fatores ou variáveis independentes, as pesquisas do tipo survey são, por sua própria natureza, estáticas em relação ao tempo, ao espaço e aos grupos populacionais atingidos. ${ }^{1}$

Desta forma, apresentam limitações cruciais para a descrição e análise de processos reconhecidamente dinâmicos como os eventos relacionados com o crescimento e desenvolvimento de crianças e os problemas correlatos de alimentação, nutrição e saúde, notadamente em populações que apresentam rápidas mudanças em seus padrões demográficos e em seus perfis de morbi-mortalidade. Este é o caso do Brasil, da grande maioria dos países da América Latina e do bloco das nações em desenvolvimento que experimentam um processo de transição epidemiológica. 2,3

Por conseguinte, a realização de estudos de coorte, de natureza observacional ou incorporando intervenções, representa uma iniciativa de instigante interesse científico e administrativo, oferecendo subsídios para uma compreensão mais adequada da situação de saúde e nutrição e uma concepção mais consistente das ações recomendadas. É dentro dessa perspectiva que foi desenvolvida a concepção desta pesquisa na Zona da Mata Meridional de Pernambuco, por se tratar de um ecossistema de configuração muito peculiar no Nordeste brasileiro, tendo a monocultura açucareira como atividade econômica dominante, imprimindo características próprias à organização política e social no meio rural e no espaço urbano. O desemprego sazonal, a fragilidade dos sistemas produtivos alternativos (fruticultura, pequenas lavouras de mandioca), o subemprego em atividades subsidiárias de comércio urbano e da prestação de serviços avulsos fazem da Zona da Mata de Pernambuco a área socialmente mais vulnerável e, portanto, mais exposta aos problemas de saúde e nutrição que resultam de condições desfavoráveis de vida.

Ao lado do enfoque metodológico, o projeto inclui o estudo de problemas praticamente inexplorados na região e no próprio país, como a deficiência de ácidos graxos essenciais e de vitamina A e E em recém-natos, rede social de apoio ao aleitamento materno, dieta complementar à alimentação infantil, fatores relacionados com o nível de hemoglobina e desenvolvimento mental e motor de crianças e outros aspectos reunidos em sub-projetos específicos.

Este artigo tem por objetivo apresentar as características sócioeconômicas e demográficas das famílias e o perfil nutricional de crianças ao nascer, residentes em áreas urbanas de quatro municípios da Zona da Mata Meridional de Pernambuco. Também são apresentados dados longitudinais do nascimento aos 12 meses de vida relativos ao aleitamento e morbidade por diarréia, e o perfil nutricional e da situação vacinal das crianças com um ano de vida.

\section{Métodos}

O estudo longitudinal consistiu de uma coorte de 652 crianças, identificadas mediante amostragem sistemática de um total de 1.909 nascidos vivos nas áreas urbanas dos municípios estudados, representando mais de $90 \%$ dos partos ocorridos no período.

As quatro cidades incluídas no estudo, Palmares, Água Preta, Catende e Joaquim Nabuco, distam cerca de 120 km do Recife, PE, Brasil, com uma população total de aproximadamente 135.000 habitantes.

$\mathrm{O}$ recrutamento das crianças foi realizado em seis maternidades, no período de setembro de 1997 a agosto de 1998. Tendo em vista a diferença do peso ao nascer entre os sexos, foram adotados diferentes pontos de corte para a seleção da coorte com base na média de peso por sexo em estudos realizados previamente na área. 4 Para cada criança do sexo masculino, nascida com peso inferior a $3100 \mathrm{~g}$, foi recrutada uma nascida logo a seguir, com peso maior ou igual a $3100 \mathrm{~g}$, enquanto para as crianças sexo feminino, o ponto de corte foi de $3000 \mathrm{~g}$, na razão de 1:1. Foram adotadas como critério de exclusão as malformações congênitas, hipóxia perinatal e gemelaridade.

Os recém-nascidos tiveram as medidas antropométricas e a idade gestacional avaliadas nas primeiras 24 horas de vida por um dos dois assistentes de pesquisa (enfermeira/nutricionista). Para avaliação da idade gestacional, foi adotado o método de Capurro et al. ${ }^{5}$ Nessa ocasião as mães foram entrevistadas, utilizando-se questionários pré-codificados com perguntas fechadas sobre as condições socioeconômicas, ambientais e demográficas das famílias, sobre as características reprodutivas maternas e da assistência ao pré-natal. As mães foram avaliadas quanto ao estado nutricional post-partum através de variáveis antropométricas (peso e altura).

O acompanhamento do aleitamento materno, da morbidade e do crescimento das crianças ao longo dos 18 meses foi realizado através de visitas domiciliares. Para os dois primeiros (aleitamento materno e morbidade por doença diarréica e infecção respi- 
ratória aguda), as visitas foram realizadas duas vezes por semana nos primeiros 12 meses e uma vez por semana dos 12 aos 18 meses, por uma equipe de 15 entrevistadoras, totalizando, aproximadamente, 68.000 visitas.

As informações sobre alimentação e morbidade eram prestadas pelas mães e incluíam: consumo de leite materno, água, chá, suco, leite artificial e outros alimentos, ocorrência de diarréia, presença de sangue nas fezes, tosse, cansaço, febre e vômito. Em caso de tosse e cansaço, a frequiência respiratória era aferida duas vezes pela visitadora, com cronômetro distribuído pelo Fundo das Nações Unidas para a Infância (UNICEF) e, em caso de febre, a temperatura era aferida com termômetro digital. Aleitamento materno exclusivo foi definido como o uso do leite materno diretamente da mama ou extraído sem a utilização de nenhum outro líquido, como água, chá e suco ou sólidos, com exceção de gotas ou xaropes de vitaminas, minerais ou medicamentos. $O$ conceito de aleitamento materno total foi utilizado para os casos em que as crianças recebiam leite materno diretamente do seio ou extraído, independentemente de estar recebendo qualquer alimento ou líquido, incluindo leite não humano.

A ocorrência da diarréia foi computada pelo total de dias com a doença e/ou pelo número de episódios, considerando-se um mínimo de três dias entre a ocorrência dos mesmos. Duas supervisoras asseguravam diariamente a qualidade das informações que eram complementadas em reuniões semanais com a equipe de campo. Quando se constatava a ocorrência das situações mencionadas acima, eram adotados critérios para encaminhamento à assistência médica.

As medidas de peso, comprimento e perímetros cefálico e torácico foram realizadas durante as visitas às residências aos 2, 4, 6, 9, 12, 15 e 18 meses de vida da criança, por duas antropometristas especialmente treinadas, utilizando equipamentos e técnicas padronizadas, obedecendo aos procedimentos recomendados pela World Health Organization (WHO), 6 totalizando cerca de 4400 visitas. Utilizaram-se balanças de 10 e $25 \mathrm{~kg}$ (Modelo MP10 e MP25, CMS, Londres, Reino Unido), com precisão de $10 \mathrm{~g}$, antropômetro de madeira com amplitude de $130 \mathrm{~cm}$ e sub-divisões de $0,1 \mathrm{~cm}$ e fita métrica de fibra de vidro com amplitude de $100 \mathrm{~cm}$ e sub-divisões de $0,1 \mathrm{~cm}$. O estado nutricional foi avaliado através dos indicadores peso/idade, comprimento/ idade e peso/comprimento, utilizando-se o ponto de corte $<-2$ escores $\mathrm{z}$ para classificar déficit nutricional. 6

Como desdobramento desta etapa diagnóstica foram implementados alguns sub-projetos de pesquisa (avaliação dos ácidos graxos e micronutrientes, rede social de apoio ao aleitamento materno, fatores determinantes do desmame e perfil da dieta complementar do lactente, fatores determinantes dos níveis de hemoglobina e do desenvolvimento mental e motor aos 12 meses) e de intervenção (impacto da estimulação psicossocial no desenvolvimento mental e motor dos 12 aos 18 meses), visando a melhoria das condições de saúde e nutrição da população materno-infantil.

\section{Processamento dos dados}

Os questionários e formulários dos subprojetos relacionados eram compostos, na sua maioria, de perguntas fechadas e pré-codificadas. A codificação ocorria no momento da coleta, sendo revista diariamente pelos supervisores de campo. No caso de haver perguntas abertas, a codificação era feita posteriormente pelo coordenador do subprojeto. A digitação dos dados foi realizada com dupla entrada, utilizando-se os softwars D-base III e Epi-info, versão 6,04 , imediatamente após a coleta da informação. Quando eram identificadas discrepâncias na digitação e na consistência das variáveis, a correção era feita de imediato. Para facilitar a análise dos dados, os bancos foram criados de maneira tal que permitissem a unificação entre os mesmos, através de uma única variável identificadora da criança.

\section{Aspectos éticos}

O projeto e os respectivos sub-projetos foram aprovados pelos Comitês de Ética em Pesquisa do Centro de Ciências da Saúde da Universidade Federal de Pernambuco. As mães eram convidadas a participar da pesquisa e a confidencialidade dos dados era garantida. A equipe de campo, durante o período de acompanhamento da criança, se dispunha a prestar qualquer esclarecimento sobre a pesquisa.

\section{Resultados}

No período de desenvolvimento da pesquisa ocorreram 2852 nascimentos nas maternidades dos quatro municípios estudados, dos quais 1909 (67\%) procediam da área urbana. O baixo peso $(<2500 \mathrm{~g})$ e o peso insuficiente (2500 - $2999 \mathrm{~g}$ ) ao nascer foram respectivamente, $5,6 \%$ e $18 \%$. A proporção de mães adolescentes foi de $32,9 \%$ e de partos cesarianos de $17,7 \%$.

Foram recrutados para o estudo 652 recémnascidos e ao final dos 12 meses de acompanhamen- 
to observou-se uma perda de 124 (19\%) crianças, sendo $111(17 \%)$ por migração para fora da área do estudo e 13 (2\%) por ocorrência de óbito. Destes, seis $(46,2 \%)$ foram registrados no período neonatal ( $<28$ dias) e sete $(53,8 \%)$ no período pós-neonatal. Cinco óbitos $(38,5 \%)$ ocorreram no domicílio e tiveram como causas básicas doenças diarréicas e infecções respiratórias. Ao término do acompanhamento (18 meses) as perdas contabilizaram 158 crianças $(24 \%)$.

A Tabela 1 apresenta informações sobre local de residência e sobre as características socioeconômicas e biológicas maternas. Verifica-se que 59,5\% das famílias tinham uma renda per capita igual ou inferior a $1 \frac{1}{2}$ salário mínimo (SM) e apenas $16,2 \%$ ganhavam mais de um SM per capita (salário mínimo $=\mathrm{R} \$ 120,00)$. O analfabetismo entre as mães foi de $18,9 \%$, e $41,3 \%$ tinham baixo nível ou nenhuma escolaridade. A maioria das mães referia menos de 25 anos $(68,7 \%)$, sendo $35,3 \%$ adolescentes. A primiparidade foi observada em $37,4 \%$ da amostra. Com relação à assistência ao pré-natal, $19,1 \%$ das mães não freqüentaram e apenas $26,1 \%$ fizeram cinco ou mais consultas. O parto cesariano foi realizado em $16,4 \%$ das mulheres. O percentual de mães fumantes durante a gravidez foi de $14,3 \%$. A altura média das mães foi de 1,56 cm (DP 6,1), encontrando-se 16,1\% com altura igual ou inferior a $150 \mathrm{~cm}$.

Quanto às condições da habitação, 65,6\% das famílias residiam em casa própria, com uma proporção de aglomeração de $33,4 \%$ de três ou mais pessoas/dormitório. A maioria das famílias dispunha de água encanada no domicílio $(84,2 \%)$, no entanto a disponibilidade de bacias sanitárias com descarga e coleta de lixo foi de apenas $57,1 \%$ e $63,3 \%$ das habitações, respectivamente. A posse de aparelho de televisão e de rádio foi observada em $83,9 \%$ e $80,2 \%$ das famílias respectivamente, no entanto, apenas $53,4 \%$ dispunham de refrigerador (Tabela 2).

Dos recém-nascidos estudados, 332 (50,9\%) eram do sexo masculino e $320(49,1 \%)$ do sexo feminino. A média de peso ao nascer foi de $3077 \mathrm{~g}$ (DP $466 \mathrm{~g}$ ), $8,7 \%$ apresentaram baixo peso e $34,7 \%$ peso insuficiente ao nascer. Do total de recém-nascidos, $31,6 \%$ tinham comprimento inferior à $48 \mathrm{~cm}$ com uma média de 48,8 cm (DP 2,2cm) e 7,7\% nasceram com idade gestacional inferior a 37 semanas (Tabela 3 ).

O tempo de duração do aleitamento materno observado do nascimento aos 12 meses de vida evidenciou uma mediana de 94 dias (quartís 25-75 = 49214), enquanto o aleitamento exclusivo praticamente não ocorreu, apresentando uma duração mediana de 0 dias (quartís 25-75 =0-3). Aproximadamente 2/3 das mães introduziram o leite artificial antes do primeiro mês de vida (mediana 22 dias; quartís 25$75=8-50$ ). A ocorrência de diarréia foi relativamente baixa, com uma mediana de apenas dois episódios/criança/ano (quartís 25-75 = 1-4) e 21,2\% não apresentaram diarréia durante o primeiro ano de vida. Apenas $66,1 \%$ das crianças aos 12 meses de vida tinham completado o esquema de vacinação. A avaliação do estado nutricional das crianças aos 12 meses de idade revelou percentual de déficit ( $<-2$ escores z) de $6,8 \%, 11 \%$ e $0,6 \%$ para os índices peso/idade, comprimento/idade e peso/comprimento, respectivamente (Tabela 4 ). 
Características socioeconômicas e biológicas maternas dos quatro municípios estudados: Palmares, Água Preta, Catende e Joaquim Nabuco. Zona da Mata Meridional de Pernambuco, 1997 a 2000.

\begin{tabular}{|c|c|c|}
\hline Variáveis & Total $(\mathrm{N}=652)$ & $\%$ ou $\bar{X} \pm d p$ \\
\hline \multicolumn{3}{|l|}{ Local de residência } \\
\hline Palmares & 357 & 54,8 \\
\hline Água Preta & 113 & 17,3 \\
\hline Catende & 123 & 18,9 \\
\hline Joaquim Nabuco & 59 & 9,0 \\
\hline \multicolumn{3}{|l|}{ Renda per capita (SM) } \\
\hline$\leq 0,25$ & 151 & 25,3 \\
\hline $0,26-0,50$ & 204 & 34,2 \\
\hline $0,51-1,0$ & 145 & 24,3 \\
\hline$>1,0$ & 97 & 16,2 \\
\hline Analfabetismo & 123 & 18,9 \\
\hline \multicolumn{3}{|l|}{ Escolaridade (anos) } \\
\hline Nenhuma & 46 & 7,1 \\
\hline $1-4$ & 222 & 34,2 \\
\hline $5-8$ & 252 & 38,9 \\
\hline$\geq 9$ & 128 & 19,8 \\
\hline \multicolumn{3}{|l|}{ Idade (anos) } \\
\hline$<20$ & 230 & 35,3 \\
\hline $20-24$ & 218 & 33,4 \\
\hline $25-29$ & 114 & 17,5 \\
\hline \multirow[t]{2}{*}{$\geq 30$} & 90 & 13,8 \\
\hline & & $22,8 \pm 6,2$ \\
\hline \multicolumn{3}{|l|}{ Paridade } \\
\hline 1 & 244 & 37,4 \\
\hline $2-3$ & 256 & 39,3 \\
\hline \multirow[t]{2}{*}{$\geq 4$} & 152 & 23,3 \\
\hline & & $2,8 \pm 2,6$ \\
\hline \multicolumn{3}{|l|}{ Pré-natal } \\
\hline Nenhum & 123 & 19,1 \\
\hline $1-4$ & 352 & 54,8 \\
\hline$\geq 5$ & 168 & 26,1 \\
\hline Cesariana & 107 & 16,4 \\
\hline Fumantes & 93 & 14,3 \\
\hline \multicolumn{3}{|l|}{ Altura $(\mathrm{cm})$} \\
\hline \multirow[t]{2}{*}{$\leq 150,0$} & 105 & 16,1 \\
\hline & & $156 \pm 6,1$ \\
\hline
\end{tabular}


Características da habitação e bens de consumo das famílias dos quatro municípios estudados: Palmares, Água Preta, Catende e Joaquim Nabuco. Zona da Mata Meridional de Pernambuco, 1997 a 2000.

\begin{tabular}{|c|c|c|}
\hline Variáveis & Total $(\mathrm{N}=652)$ & $\%$ \\
\hline \multicolumn{3}{|l|}{ Condição da habitação } \\
\hline Própria & 428 & 65,6 \\
\hline Alugada/outros & 224 & 34,4 \\
\hline \multicolumn{3}{|c|}{ Aglomeração(nºpessoas/dormitório) } \\
\hline$<2$ & 145 & 22,2 \\
\hline $2-3$ & 289 & 44,3 \\
\hline$\geq 3$ & 218 & 33,4 \\
\hline Abastecimento d'água & 549 & 84,2 \\
\hline \multicolumn{3}{|l|}{ Sanitário } \\
\hline Com descarga & 372 & 57,1 \\
\hline Sem descarga & 204 & 31,3 \\
\hline Nenhum & 76 & 11,7 \\
\hline Coleta de lixo & 413 & 63,3 \\
\hline Iluminação elétrica & 646 & 99,1 \\
\hline Televisão & 547 & 83,9 \\
\hline Rádio & 523 & 80,2 \\
\hline Refrigerador & 348 & 53,4 \\
\hline Fogão & 629 & 96,5 \\
\hline
\end{tabular}

Tabela 3

Características das crianças ao nascer dos quatro municípios estudados: Palmares, Água Preta, Catende e Joaquim Nabuco. Zona da Mata Meridional de Pernambuco, 1997 a 2000.

\begin{tabular}{|c|c|c|}
\hline Variáveis & $(\mathrm{N}=652)$ & $\%$ ou $\bar{X}+d p$ \\
\hline \multicolumn{3}{|l|}{ Sexo } \\
\hline Masculino & 332 & 50,9 \\
\hline Feminino & 320 & 49,1 \\
\hline \multicolumn{3}{|c|}{ Peso ao nascer $(\mathrm{g})$} \\
\hline$<2.500$ & 57 & 8,7 \\
\hline $2500-2999$ & 226 & 34,7 \\
\hline $3000-3499$ & 247 & 37,9 \\
\hline \multirow[t]{2}{*}{$\geq 3500$} & 122 & 18,7 \\
\hline & & $3077 \pm 466$ \\
\hline \multicolumn{3}{|c|}{ Comprimento $(\mathrm{cm})$} \\
\hline$<48,0$ & 206 & 31,6 \\
\hline $48,0-49,9$ & 245 & 37,6 \\
\hline \multirow[t]{2}{*}{$\geq 50,0$} & 201 & 30,8 \\
\hline & & $48,8 \pm 2,2$ \\
\hline \multicolumn{3}{|c|}{ Idade gestacional (semanas) } \\
\hline$<37$ & 50 & 7,7 \\
\hline
\end{tabular}


Características das crianças no primeiro ano de vida dos quatro municípios estudados: Palmares, Água Preta, Catende e Joaquim Nabuco. Zona da Mata Meridional de Pernambuco, 1997 a 2000.

\begin{tabular}{|c|c|c|}
\hline Variáveis & $(N=652)$ & $\operatorname{Md}\left(Q_{25}-Q_{75}\right)$ ou $\%$ \\
\hline \multicolumn{3}{|l|}{ Nascimento - 12 meses } \\
\hline \multicolumn{3}{|l|}{ Aleitamento materno (dias) } \\
\hline Exclusivo & & $0(0-3)^{*}$ \\
\hline Introdução leite artificial & & $22(8-50) *$ \\
\hline Total & & $94(49-214) *$ \\
\hline \multicolumn{3}{|l|}{ Diarréia (episódios) } \\
\hline Nenhum & 138 & 21,2 \\
\hline $1-2$ & 233 & 35,7 \\
\hline $3-5$ & 188 & 28,8 \\
\hline \multirow[t]{2}{*}{$\geq 6$} & 93 & 14,3 \\
\hline & & $2(1-4) *$ \\
\hline Aos 12 meses & $(n=528)$ & \\
\hline \multicolumn{3}{|l|}{ Vacinação } \\
\hline Esquema completo & 349 & 66,1 \\
\hline Esquema incompleto & 97 & 18,4 \\
\hline Sem informação & 82 & 15,5 \\
\hline \multicolumn{3}{|l|}{ Peso/ldade (escore z) } \\
\hline$<-2$ & 36 & 6,8 \\
\hline$-2 a<-1$ & 122 & 23,1 \\
\hline$\geq-1$ & 370 & 70,1 \\
\hline \multicolumn{3}{|l|}{ Comprimento/ldade (escore z) } \\
\hline$<-2$ & 58 & 11,0 \\
\hline$-2 a<-1$ & 131 & 24,8 \\
\hline$\geq-1$ & 339 & 64,2 \\
\hline \multicolumn{3}{|l|}{ Peso/Comprimento (escore $z$ ) } \\
\hline$<-2$ & 3 & 0,6 \\
\hline$-2 a<-1$ & 53 & 10,0 \\
\hline$\geq-1$ & 472 & 89,4 \\
\hline
\end{tabular}

* $\mathrm{Md}\left(\mathrm{Q}_{25}-\mathrm{Q}_{75}\right)=$ Mediana (Quartil $25-$ Quartil $\left._{75}\right)$

\section{Discussão}

O desenvolvimento do presente estudo surgiu da necessidade de melhor compreender os aspectos relativos ao crescimento e desenvolvimento infantis em populações da região Nordeste que concentram um grande contingente da população carente do país, além da tentativa de contribuir para a elaboração de novas estratégias de intervenção na área materno-infantil.
Outro objetivo diz respeito à possibilidade de realização de estudos longitudinais em áreas pobres de países em desenvolvimento como o Brasil, onde as perdas durante o acompanhamento dificultam a análise dos dados. Entretanto, o estudo foi realizado apenas com a população urbana em virtude das dificuldades inerentes à população rural no que diz respeito a sua dispersão espacial na área do estudo, bem como o intenso processo migratório do ciclo cultivo/colheita da cana-de-açúcar. Ao término do 
primeiro ano de vida, $81 \%$ das crianças permaneciam no estudo, reduzindo-se para $76 \%$ aos 18 meses de vida, resultados bem semelhantes aos encontrados em Pelotas por Victora et al.,1 e considerados satisfatórios.

O perfil demográfico e epidemiológico da população revela um quadro de transição, com um número médio de 2,8 filhos por mulher quando comparado à média de $1980(6,13)$ e $1984(4,96)$ para a região Nordeste.13 A prevalência do baixo peso ao nascer (BPN) declinou de 9,2\% em 199114,15 para 5,6\% na área urbana do estudo, representando uma diminuição de 39\%. O menor número de filhos/mulher, o maior consumo de alimentos básicos pela população de baixa renda e maior cobertura da assistência pré-natal e ao parto podem estar associados a esses achados. 16 Outro aspecto que chama a atenção é a redução de fumantes em $16,4 \%$, durante a gravidez, quando comparado com estudos realizados nesta área, nos anos 1992/1994, com percentuais de 26\%.14,15

No que diz respeito ao acompanhamento da morbidade e do perfil alimentar das crianças, o presente estudo, com duas visitas domiciliares semanais durante o primeiro ano de vida e uma visita semanal dos 12 aos 18 meses, é único no Brasil. A principal vantagem desse acompanhamento realizado com curtos intervalos entre as visitas foi de se obter com maior fidedignidade as informações, reduzindo o provável viés recordatório.

A avaliação do perfil alimentar foi complementada com estudos transversais sobre o padrão alimentar, através do método recordatório de 24 horas aos 2, 4, 6, 9 e 12 meses de vida, o que possibilitará uma maior compreensão do processo de introdução dos alimentos do desmame que desempenham, provavelmente, um papel importante no perfil epidemiológico das carências nutricionais nos menores de dois anos.

A supervisão de campo regular e as avaliações quinzenais, com todos os membros da equipe, tinham como objetivo corrigir problemas e reforçar e manter a qualidade técnica das informações. No conjunto, essas atividades possibilitaram uma maior fidedignidade das informações relatadas pelas mães e/ou responsáveis.

Neste estudo, a análise do crescimento infantil terá como destaque as crianças nascidas com peso insuficiente ao nascer ( $2500-2999 \mathrm{~g})$, tanto pela sua significativa prevalência (18\%) na área estudada, como por ser pouco referenciado na literatura. Considerando que nos dois primeiros anos de vida as crianças experimentam um ritmo acelerado de crescimento, a identificação de fatores que influenciam esse processo poderá contribuir para a implemen- tação de ações visando o crescimento adequado.

A incidência da diarréia foi considerada pouco expressiva nos menores de 12 meses, com uma mediana de dois episódios/criança/ano e uma prevalência de déficit peso/idade e comprimento/idade (<-2 escores z), respectivamente $6,8 \%$ e $11 \%$ aos 12 meses de vida, semelhante aos observados para o interior do estado de Pernambuco $(5,2 \%$ e $13,5 \%$, respectivamente). 16

Apesar desse quadro aparentemente favorável, as condições socioeconômicas apontam para uma população de baixo poder aquisitivo, com $60 \%$ da renda per capita das famílias $\leq 0,50 \mathrm{SM}, 41,3 \%$ das mães com menos de quatro anos de escolaridade e uma taxa de analfabetismo de 19\%. Embora a população disponha de boa cobertura em termos de acesso à rede elétrica e abastecimento d'água $(99 \%$ e $84 \%$, respectivamente), observou-se que $43 \%$ e $47 \%$ das famílias não contam com rede de esgotos sanitários e serviços de coleta de lixo, respectivamente. Com relação à assistência à saúde, $19 \%$ das mães não realizaram o pré-natal, 55\% apresentaram um número de consultas inferior ao preconizado e apenas $66 \%$ das crianças tinham completado o esquema de vacinação aos 12 meses de idade. Dos 13 óbitos notificados durante o primeiro ano de vida, cinco $(38,5 \%)$ ocorreram no domicílio, fato sugestivo de uma assistência ainda insatisfatória das doenças mais prevalentes na infância, tendo em vista que as causas básicas do óbito foram diarréias e infecções respiratórias agudas (IRA).

Embora o aleitamento materno tenha sido iniciado pela quase totalidade das mães (98\%), o desmame ocorre precocemente, com uma duração mediana do aleitamento materno total de 94 dias e do aleitamento materno exclusivo de 0 dias. Esses resultados preliminares motivaram a implementação de um projeto de incentivo ao aleitamento materno nos quatro municípios estudados, envolvendo os profissionais de saúde que atuam nas maternidades e uma equipe de campo treinada no manejo e suporte ao aleitamento materno através de visitas domiciliares, durante os seis primeiros meses de vida. A idéia central deste projeto foi o desenvolvimento de atividades possíveis de serem implementadas no Programa de Agentes Comunitários de Saúde (PACS), visando o apoio ao binômio mãe/filho na prática do aleitamento materno exclusivo.

Outro aspecto de interesse central se refere ao desenvolvimento infantil, especialmente em populações carentes, que praticamente não contam com cuidados nessa área, impossibilitando uma maior compreensão e controle do processo crescimento/desenvolvimento, tanto por parte dos profissionais de 
saúde, como pelas famílias. A elaboração de um programa de estimulação psicossocial nessa comunidades possibilitou uma maior valorização desses processos, em crianças sem comprometimento biológico e que vivem em ambientes socialmente desfavoráveis. A experiência acumulada e os resultados dessa intervenção poderão contribuir para uma melhor performance dos profissionais que atuam na comunidade, através do PACS e Programa Saúde da Família (PSF), contribuindo desta forma para uma maior interação entre mãe e/ou responsável e a criança.

As maternidades dos municípios envolvidos nestes projetos vêm recebendo comprovações que evidenciam uma repercussão positiva no enfoque dos problemas identificados, mediante ações imediatas tanto dos profissionais como dos administradores locais de serviços de saúde. O retorno dos resultados à comunidade vem ocorrendo de forma efetiva, seja através de artigos na imprensa local, de emissoras de rádio nas quais se tem a oportunidade de interlocução com a comunidade e, ainda, através de eventos públicos, como a "Semana Pró-Aleitamento Materno", com ampla mobilização nos quatro municípios estudados. dos. 8,17

Alguns resultados também já foram publica-

\section{Agradecimentos}

Às famílias participantes, aos 22 trabalhadores de campo, à equipe de processadores e digitadores dos dados, ao staff das seis maternidades da área, ao Hospital Regional dos Palmares da Secretaria de Saúde do Estado de Pernambuco, a CAPES e ao Conselho Britânico. Ao Instituto Nacional de Alimentação e Nutrição do Ministério da Saúde, (INAN/MS - Processo $\left.n^{\circ} 25350.001472 / 96\right)$ pelo suporte financeiro.

\section{Referências}

1. Victora CG, Barros FC, Martines JC, Béria JU, Vaughan JP. Estudo longitudinal das crianças nascidas em $1982 \mathrm{em}$ Pelotas, RS, Brasil. Rev Saúde Pública 1985; 19: 58-68.

2. Monteiro CA. Velhos e novos males da saúde no Brasil: a evolução do país e de suas doenças. São Paulo: Hucitec; 1995.

3. Rissin A, Batista Filho M, Lima MC, Costa MJC. Estado de nutrição de crianças nordestinas no advento dos anos 90: associação com algumas variáveis biológicas. Rev IMIP 1999; 13: 109-13.

4. Ashworth A, Morris SS, Lira PIC. Postnatal growth patterns of full-term low birth weight infants in Northeast Brazil are related to socioeconomic status. J Nutr 1997; 127: 1950-6.

5. Capurro H, Konicheszky S, Fonseca D, Caldeyro-Garcia R. A simplified method for diagnosis of gestational age in the newborn infant. J Pediatr 1978; 93: 120-2.

6. WHO (World Health Organization). Physical status: the use and interpretation of anthropometry: report of an Expert
Committee. Geneva: The Organization; 1995. (Technical Report Series, 854).

7. Oppenheim AN. Questionnaire design, interviewing and attitude measurement. London: Printer; 1997.

8. Marques NM, Lira PIC, Lima MC, Silva NL, Batista Filho M, Huttly SRA, Ashworth A. Breastfeeding and early weaning practices in Northeast Brazil: a longitudinal study. Pediatrics [serial on line] 2001; 108: 66. Available from: http://www.pediatrics.org/cgi/content/full/108/ 4/e66. [20/08/2002]

9. Nelson M, Bigham SA. Assessment of food consumption and nutrient intake. In: Margets BM, Nelson M, editor. Design concepts in nutritional epidemiology. New York: Oxford University Press; 1996. p. 123-69.

10. Philippi ST, Szarfarc SC, Latterza AR. Virtual nutri [computer program] versão 1.0, for windows. São Paulo: Universidade de São Paulo; 1996.

11. Bayley N. Bayley scale of infant development. Berkley: The Psychological Corp; 1969. 
12. Caldwell BM. Instruction manual: home inventory for infants. Little Rock: University of Arkansas; 1975.

13. FIBGE (Fundação Instituto Brasileiro de Geografia e Estatística), UNICEF (Fundo das Nações Unidas para a Infância), OPAS (Organização Pan-Americana da Saúde). Perfil estatístico de crianças e mães no Brasil: a situação da fecundidade; determinantes gerais e características da transição recente. Rio de Janeiro: A Fundação; 1988

14. Lima MC, Ismail S, Ashworth A, Morris SS. Influence of heavy agricultural work during pregnancy on birthweight in Northeast Brazil. Int J Epidemiol 1999; 28: 469-74.

15. Lira PIC, Ashworth A, Morris SS. Low birth weight and morbidity from diarrhea and respiratory infection in Northeast Brazil. J Pediatr 1996; 128: 497-504.
16. INAM (Instituto Nacional de Alimentação e Nutrição), IMIP (Instituto Materno Infantil de Pernambuco), Universidade Federal de Pernambuco [Departamento de $\mathrm{Nu}$ trição], Recife. Secretaria de Saúde. II Pesquisa Estadual de Saúde e Nutrição: saúde, nutrição, alimentação e condições socioeconômicas no Estado de Pernambuco. Recife: Ministério da Saúde; 1998.

17. Barros MFA, Leger CL, Lira PIC, Lima MC, Carbonneau M-A, Descomps B, Alessio MLM. Cord blood essential fatty acid and alpha-tocopherol in full-term newborns in a Northeast Brazil area. Int J Vitam Nutr Res 2002; 72: 155-60.

Recebido em 18 de janeiro de 2003

Versão final reapresentada em 6 de abril de 2003

Aprovado em 9 de julho de 2003 